\title{
Complex evaluation of the quality of introduced and local varieties of Virginia flue-cured tobacco in Bulgaria
}

\author{
Nikolay Nikolov ${ }^{1}$, Violeta Nikolova ${ }^{1}$, and Venelina Popova, ${ }^{2, *}$ \\ ${ }^{1}$ Tobacco and Tobacco Products Institute, Agricultural Academy, Markovo village, Rodopi district, Bulgaria \\ ${ }^{2}$ Department of Tobacco, Sugar, Vegetable and Essential Oils, University of Food Technologies, Plovdiv, Bulgaria
}

\begin{abstract}
The integrated influence of trade, technological and production fundamentals that shape the market of flue-cured Virginia (FCV) tobacco in Bulgaria objectifies the need of systematic surveys on the quality of the tobacco varieties represented in the country. Therefore, current investigation regarded the quality level of PVH19 and Niki tobacco varieties in the regions of South and North Bulgaria (labeled conditionally as "local"), as well as that of two introduced varieties, H9 (Hungary) and V (Greece). The basic indicators of FCV tobacco quality were analyzed (leaf and smoke chemical composition, external leaf quality elements, smoking quality), and the final evaluation was completed by determining the complex "quality index score". The results from the study revealed differences in tobacco quality formation, both on a region and variety basis, in terms of leaf and smoke chemical composition, expert and smoking assessment. The final rating of the studied varieties by the complex evaluation procedure was as follows: the best quality level was found for the $\mathrm{H} 9$ variety introduced for the region of Plovdiv, followed by PVH19 variety from the same region, the next were PVH19 (Parvomay) and V (Central North Bulgaria) varieties, and the lowest rated was the local Niki variety (Central North Bulgaria).
\end{abstract}

\section{Introduction}

The market tendencies in the production of Virginia fluecured (FCV) tobacco established in the last years have been substantiated by a number of specific interrelated mechanisms, some of which include: the production and realization of tobacco batches through a considerable diversity in the varietal structure; the buyers' demand for predominantly stripped and blended material; the realization of negotiated samples of tobacco strips in a buyer-defined percentage combination of individual components; the direct incorporation of big-scale component blends with specific "geographical" casing in brands' portfolios; the prospects of tobacco use in final products with lower consumer status, and others. Those tendencies underline the necessity of establishing homogenous tobacco batches, regardless of the effective varietal configuration and/or the production region. The typification of the produced tobaccos on a quality basis is also necessary for their arrangement in groups, depending on the respective designation, as individual components in the tobacco blends for different smoking products.

Many studies on those mechanisms had indicated that the accomplishment of certain tobacco quality attributes was defined mainly by the inherent potential (genetics) of the respective variety, the applied agricultural practices, the maturity status of harvested leaves, and the curing regime parameters, as well as by other specific factors [110]. Furthermore, it had been ascertained that the application of differentiated agro-technical activities could be a means for directing the tobacco production towards the achievement of the preferred technological characteristics $[9,11,12]$.

The establishment of effective market mechanisms in the tobacco sector imposed the detailed comprehension of tobacco varieties' potential, as well as the introduction of varieties with valuable biological and economic qualities, which would allow tobacco producers and dealers to realize batches and blends consisting of different quality groups.

The above-described market, technological and production realities, which currently shape the status of large-leaf tobaccos in Bulgaria, objectify the need for annual crop surveys and the relevant grouping of the producing regions with different tobacco quality level depending on the market demand for the respective crop, as well as the compiling of batches of characteristic leaf material offered on the market for different product blends. Therefore, the objective of this study was the evaluation of the quality indices and the patterns of complex quality formation in introduced and local FCV tobacco varieties from different production regions in Bulgaria.

\footnotetext{
* Corresponding author: vpopova2000@abv.bg
} 


\section{Materials and methods}

\subsection{Plant material}

The study was conducted in the period 2017 - 2018 and included two groups of FCV tobacco varieties previously cultivated (labelled conditionally as "local" in the study, although not locally selected) and introduced (international) - produced in different regions of South Bulgaria and North Bulgaria tobacco areas, as follows:

a) Region Plovdiv (South Bulgaria area) - a local variety (PVH19, mass-produced) and an introduced variety (H9, origin: Hungary);

b) Region Parvomay (South Bulgaria area) - a local variety (PVH19);

c) Region Central North Bulgaria (North Bulgaria area) - a local variety (Niki) and an introduced variety (V, origin: Greece).

Tobacco production followed the established agricultural practices in the respective region; leaf harvesting and curing was according to the standard technology for FCV tobacco [13]. The analytical samples were formed by selecting the characteristic FCV tobacco stalk position, cutters (C) [14]. In compliance with the objectives of the study, the farmer-supplied bulk samples were pre-sorted (equalized) in order to secure uniform material for the analysis of the varieties.

\subsection{Evaluation procedure}

Tobacco samples were assessed consecutively in terms of the following groups of quality indices:

\subsubsection{Chemical composition of tobacco leaf and tobacco smoke}

The basic chemical characteristics of FCV tobacco were determined according to the standard methods, as follows: total alkaloids, as nicotine [15], reducing sugars [16], total nitrogen [17], mineral matter (ash) [18], potassium content, as $\mathrm{K}_{2} \mathrm{O}$ [19], and total $n$-hexane extract (on a "Soxtec HT-6" extractor, Tecator Inc., USA).

Additionally, the two ratios indicative of FCV tobacco quality and balance, total nitrogen/nicotine and reducing sugars/nicotine, were calculated from the analytical data. All analyses were performed in triplicate and the data were presented as mean values \pm standard deviation $(n=3)$.

Smoke nicotine (mg/cig) and tar (mg/cig) values were derived from the regression models established for FCV tobacco [20]. In those models, data were relevant to a standard filter-tipped cigarette, with $84 \mathrm{~mm}$ total length, $7.9 \mathrm{~mm}$ diameter, and $21 \mathrm{~mm}$ filter plug length $(3 / 35000 \mathrm{Y}$ mono-acetate filter).

\subsubsection{Assessment of differences in chemical data}

The assessment and interpretation of the detected differences between the numerical values of the chemical indices of the varieties (nicotine, reducing sugars, total nitrogen, ash, smoke nicotine, and tar), as well as the value range for defining the respective chemical index level (low, medium, high), were according to the criteria specified previously $[21,22]$. Thus, the samples within a series were considered different if the deviation between the respective values was within or above the range cited.

\subsubsection{Leaf quality elements (expert assessment)}

The assessment of leaf quality elements was carried out by a five-member expert panel, with the task of completing an integrated valuation of all external leaf characteristics expressive of tobacco quality [23]. The direct comparison method was applied, on coded samples (blind assessment test).

The unanimity of expert rankings was statistically tested either by the critical ratio number $(C R N$, in the paired comparison of two samples) or by the coefficient of concordance $(W)$ and the $F$-test (in the paired comparison of three or more samples). The calculated $C R N$ and $W$ values were considered statistically significant if exceeding 1.96 and 0.50 , respectively (at a $95 \%$ probability level), i.e. the individual expert rankings could be considered unanimous [24]

\subsubsection{Smoking profile (smoking assessment)}

The assessment of tobacco smoke perception was completed by a five-member smoking panel, on coded samples (representing laboratory-made single-tobacco non-filter cigarettes), in a full combination of the varieties in the paired-comparison test. The statistical processing of the individual results was the same as in the expert assessment.

\subsubsection{Complex evaluation of tobacco quality level}

The final evaluation of the tobaccos in the study was based on the results from the assessment of the basic indices defining tobacco quality, i.e. leaf chemical composition (indices selected as characteristic of the tobacco type), smoke chemical composition, expert and smoking assessment. Each sample was ranked according to the respective index value, accounting for its positive or negative correlation with tobacco quality. The coefficients of importance (relative weight) of those indices were defined in advance by the expert panel. The final rating of the varieties was obtained by calculating the individual values of a "quality index" and the total score of quality indices; the lower index score indicated higher rating, and respectively, better quality $[6,21,22]$.

\section{Results and discussion}

\subsection{Chemical composition of FCV tobacco varieties}

Data from the analysis of the basic chemical indices of the studied FCV tobacco varieties (leaf and smoke composition) are presented in Table 1 . 
Table 1. Indices of the chemical composition of the FCV tobacco varieties in the study

\begin{tabular}{|c|c|c|c|c|c|c|c|c|c|}
\hline \multirow{3}{*}{ Region } & \multirow{3}{*}{ Variety } & \multicolumn{8}{|c|}{ Index } \\
\hline & & \multicolumn{6}{|c|}{ Tobacco } & \multicolumn{2}{|c|}{ Smoke } \\
\hline & & $\operatorname{Nic}^{1}(\%)$ & $\mathrm{RS}^{2}(\%)$ & RS/Nic & $\mathrm{TN}^{3}(\%)$ & TN/Nic & Ash (\%) & $\begin{array}{c}\text { Nic } \\
\text { (mg/cig) }\end{array}$ & $\begin{array}{c}\text { Tar } \\
\text { (mg/cig) }\end{array}$ \\
\hline Plovdiv & PVH19 & $1.77 \pm 0.01$ & $19.40 \pm 0.18$ & 10.96 & $1.47 \pm 0.01$ & 0.83 & $8.53 \pm 0.08$ & 1.43 & 22.13 \\
\hline Plovdiv & H9 & $2.11 \pm 0.02$ & $23.60 \pm 0.22$ & 11.18 & $1.45 \pm 0.01$ & 0.69 & $8.04 \pm 0.08$ & 1.78 & 19.56 \\
\hline Parvomay & PVH19 & $1.23 \pm 0.01$ & $18.20 \pm 0.18$ & 14.80 & $1.51 \pm 0.01$ & 1.23 & $10.76 \pm 0.09$ & 0.97 & 22.48 \\
\hline $\begin{array}{c}\text { Central North } \\
\text { Bulgaria }\end{array}$ & Niki & $1.17 \pm 0.01$ & $28.40 \pm 0.24$ & 24.27 & $0.75 \pm 0.01$ & 0.64 & $9.06 \pm 0.09$ & 0.92 & 18.87 \\
\hline $\begin{array}{l}\text { Central North } \\
\text { Bulgaria }\end{array}$ & V & $1.09 \pm 0.01$ & $26.90 \pm 0.24$ & 24.68 & $1.00 \pm 0.01$ & 0.92 & $8.27 \pm 0.08$ & 0.87 & 18.74 \\
\hline
\end{tabular}

${ }^{1} \mathrm{Nic}$ - nicotine; ${ }^{2} \mathrm{RS}$ - reducing sugars; ${ }^{3} \mathrm{TN}$ - total nitrogen.

The interpretation of the data about the chemical indices of the individual samples, as stated above, was focused mainly on the indicators referred to as characteristic for FCV tobacco, i.e. nicotine and reducing sugars content, and the proportions total nitrogen/nicotine and reducing sugars/nicotine. In general, good quality FCV tobacco is associated with nicotine content above $1.8 \%$, total nitrogen/nicotine ratio between 0.6 and 1.0 (thus suggesting prevalence of nicotine in the total content of nitrogenous compounds), limited reducing sugars content (between $15 \%$ and $20 \%$ ), and relatively lower reducing sugars/nicotine ratio (between 7 and 12), the latter values being common to the high quality ("typical") FCV tobacco and indicative of better balance in the smoking profile [25].

\subsubsection{South Bulgaria (regions Plovdiv and Parvomay).}

As seen from Table 1, the nicotine content in the tobacco varieties from the region of Plovdiv (PVH19 and H9) was definitely higher than that in the local variety (PVH19) from the region of Parvomay; the indices total nitrogen/nicotine and reducing sugars/nicotine were more favorable (more balanced), as well. The rest of the chemical indices, total nitrogen and ash contents, showed no significant variation between the varieties and the regions. Consistently, the highest concentration of nicotine in the tobacco smoke was found in the introduced H9 variety from the region of Plovdiv, and the lowest - in PVH19 variety from Parvomay. The predicted tar contents followed an inverse order; the highest levels were registered in PVH19 variety, regardless of the production region, thus discriminating from the introduced H9 variety with the lowest tar level.

Those results suggested that the local PVH19 variety from the region of Plovdiv established definitely better chemical indices in the series, which were common to those of the "typical" FCV tobaccos on a worldwide basis, e.g. higher leaf and smoke nicotine $(1.77 \%$ and 1.43 $\mathrm{mg} / \mathrm{cig}$, respectively) and lower total nitrogen/nicotine $(0.83)$ and reducing sugars/nicotine (10.96) ratios.

\subsubsection{North Bulgaria (region Central North Bulgaria).}

According to the adopted criteria for the interpretation of differences in chemical indices values, there were no significant differences between the regarded tobaccos (the local Niki and the introduced V varieties), both in terms of the individual experimental results and the calculated chemical ratios (Table 1). Therefore, the data from the chemical analysis of the varieties in the Central North Bulgaria region were suggestive of a relatively pronounced tobacco uniformity.

\subsection{Expert assessment of FCV tobacco varieties}

In the first stage of the expert assessment procedure, the studied tobacco varieties were rated by a direct comparison within the respective tobacco producing area.

The results obtained from those rating series (Fig. 1) created the following order for the varieties in South Bulgaria tobacco area: the best rated was H9 variety (origin: Hungary, region Plovdiv), followed by PVH19 (Parvomay) and PVH19 (Plovdiv); the expert ranking was unanimous and significant $(W=0.84)$. In turn, the expert assessment of the two varieties from the North Bulgaria tobacco area rated the introduced $\mathrm{V}$ variety (origin: Greece) as better than the local Niki variety.

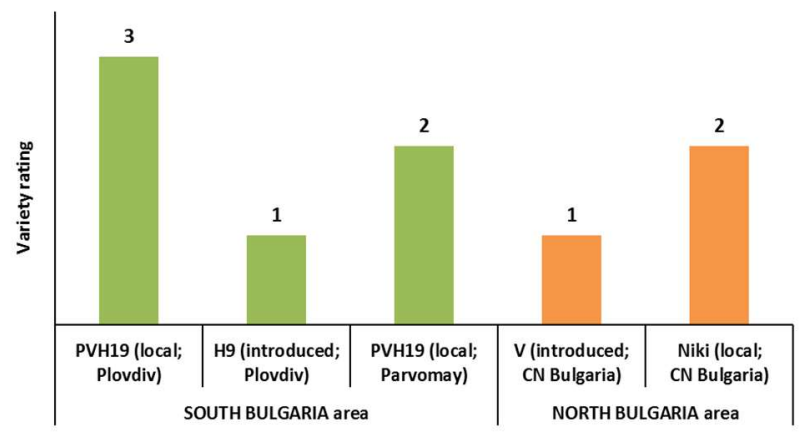

Fig. 1. Rating of FCV varieties from South Bulgaria (left) and North Bulgaria (right) areas by expert assessment 
Table 2. Expert assessment of the FCV tobacco varieties from South and North Bulgaria regions

\begin{tabular}{|c|c|c|c|c|c|}
\hline \multirow{3}{*}{ Expert (i) } & \multicolumn{5}{|c|}{ Variety rank $(j)$} \\
\hline & PVH19 & H9 & PVH19 & $\mathbf{V}$ & Niki \\
\hline & \multicolumn{2}{|c|}{ Plovdiv } & Parvomay & \multicolumn{2}{|c|}{ Central South Bulgaria } \\
\hline 1 & 5 & 1 & 2 & 3 & 4 \\
\hline 2 & 5 & 1 & 2 & 3 & 4 \\
\hline 3 & 4 & 2 & 1 & 3 & 5 \\
\hline 4 & 5 & 1 & 2 & 3.5 & 3.5 \\
\hline 5 & 5 & 1 & 4 & 2 & 3 \\
\hline$\sum x_{i j}$ & 24 & 6 & 11 & 14.5 & 19.5 \\
\hline Relative ranking coefficient & 0.32 & 0.08 & 0.15 & 0.19 & 0.26 \\
\hline Ranking coefficient & 0.25 & 1.00 & 0.55 & 0.41 & 0.31 \\
\hline Rating & 5 & 1 & 2 & 3 & 4 \\
\hline
\end{tabular}

Considering the decisive role of the expert assessment of tobacco quality for tobacco farmers' revenue and the relevance of paralleling the local mass-produced varieties with the introduced ones in terms of leaf quality elements on a wider (i.e. producing country) basis, a direct comparison of all FCV tobaccos in the study was conducted (Table 2).

The processing of the individual panelist data in Table 2 revealed significant differences between the varieties $(W=0.79)$ and the obtained rating by the expert assessment could be accepted as valid. Thus, the best rated by external leaf quality elements was the $\mathrm{H} 9$ variety from the region of Plovdiv, followed in descending order by PVH19 (Parvomay), V and Niki (Central South Bulgaria), and PVH19 (Plovdiv).

\subsection{Smoking assessment of FCV tobacco varieties}

The ranking of the studied varieties in the smoking assessment tests was completed according to the integrated perception of the individual attributes of tobacco smoke and the overall smoking profile (aroma, taste and strength). The same scheme adopted for the expert assessment was applied in the assessment procedure by the smoking panel.

The comparison in the first step of the procedure, between the varieties within the South Bulgaria area (regions Plovdiv and Parvomay) and the North Bulgaria area (Central South Bulgaria region), respectively, showed no significant variation in variety ranking by smoking perception. However, the individual direct comparison of the introduced varieties (V and H9) with the local varieties (PVH19 and Niki), in a fullcombination comparison scheme, proved statistically significant differences in the smoking profiles in each series, with coefficient of concordance $(W)$ values between 0.84 and 1.00 (at 95\% confidence level). Those results rated the tobaccos from the two South Bulgaria regions (varieties PVH19 and $\mathrm{H} 9$ ) as better, in terms of smoking quality, than the tobaccos originating from the North Bulgaria area.

Those data were the basis for completing the final rating of the studied varieties by the smoking panel and the summarized results from the smoking assessment procedure are presented in Table 3.

The differences between the compared tobacco varieties were statistically significant $(W=0.88$, at $95 \%$ probability level); therefore, the individual ranking was unanimous and the final rating of the varieties could be assumed valid, as follows: the best in terms of the smoking profile was the local PVH19 variety (region Plovdiv), followed by the introduced $\mathrm{H} 9$ variety from the same region, then the rest of the varieties, PVH19 (Parvomay), V (Central North Bulgaria) and Niki (Central North Bulgaria).

Table 3. Smoking assessment of the FCV tobacco varieties from South and North Bulgaria regions

\begin{tabular}{|c|c|c|c|c|c|}
\hline \multirow{2}{*}{ Expert $(\boldsymbol{i})$} & \multicolumn{5}{|c|}{ Variety rank $(\boldsymbol{j})$} \\
\cline { 2 - 6 } & PVH19 & H9 & PVH19 & V & Niki \\
\cline { 2 - 6 } & \multicolumn{2}{|c|}{ Plovdiv } & 2 & Parvomay & \multicolumn{2}{c|}{ Central South Bulgaria } \\
\hline 1 & 1 & 1 & 2.5 & 4 & 5 \\
2 & 2.5 & 1 & 3 & 4 & 5 \\
3 & 2 & 3 & 2 & 4 & 5 \\
4 & 1 & 2 & 3 & 4 & 5 \\
5 & 1 & 9 & 13.5 & 20 & 25 \\
\hline$\sum x_{i j}$ & 7.5 & 0.12 & 0.18 & 0.27 & 0.33 \\
\hline Relative ranking coefficient & 0.10 & 0.83 & 0.56 & 0.38 & 0.30 \\
\hline Ranking coefficient & 1.00 & $\mathbf{2}$ & $\mathbf{3}$ & $\mathbf{4}$ & $\mathbf{5}$ \\
\hline Rating & $\mathbf{1}$ & & & & 4 \\
\end{tabular}




\subsection{Complex evaluation of FCV tobacco varieties}

In compliance with the objectives of the study, the concluding step in the procedure aimed at the evaluation of the complex quality level of the FCV tobacco varieties from the regarded regions. As stated previously in the methodology section, the complex evaluation of the FCV varieties in the study incorporated the basic indices of tobacco quality, both objective (by chemical analysis) and sensory (by expert and smoking assessment). The ranks assigned to the varieties with regard to the respective indices of leaf and smoke chemical composition reflected the results from the preceding steps in the evaluation procedure, and in particular - the interpretation of the individual index values and their correlation, positive or negative, with tobacco quality. The ranking orders from the expert and smoking assessments, respectively, were applied directly.

The rating obtained from the calculation of the quality index values and their total score in the complex evaluation of the FCV varieties within each of the two tobacco producing areas (South Bulgaria and North Bulgaria, respectively) is presented on Fig. 2.

As seen from Fig. 2, the results for the tobaccos produced in the regions of South Bulgaria area revealed better complex quality characteristics in the introduced $\mathrm{H} 9$ variety (origin: Hungary) compared with the local PVH19 variety; in turn, the local and the introduced varieties from Central North Bulgaria region obtained equal quality indices and respectively - equal ranks, thus suggesting of uniformity and common profile of the quality level of the studied tobaccos from that region.

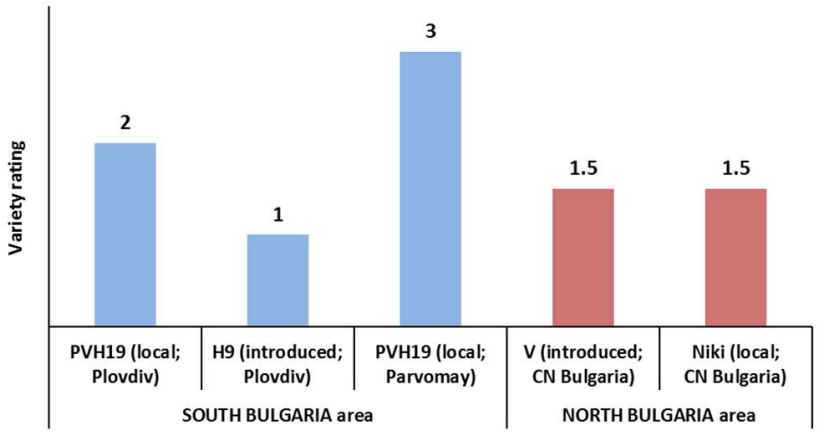

Fig. 2. Rating of the FCV varieties from South Bulgaria (left) and North Bulgaria (right) areas by complex evaluation

The summarized matrix of the complex evaluation and the resultant final rating of the FCV tobacco varieties in the study are presented in Table 4.

As seen from the calculated quality index scores in the complex evaluation matrix, the most favorable complex quality level was demonstrated by the introduced H9 variety (origin: Hungary) from the region of Plovdiv, followed by the local PVH19 variety produced in the same region. The local PVH19 variety from the region of Parvomay and the introduced V variety (origin: Greece) from the Central North Bulgaria region were rated equally, sharing the third and the fourth position, while the local variety Niki (region Central North Bulgaria) was the last in that descending quality line. Those differences reflected the combined influence of the genetic (tobacco variety) and the environmental (region) factors, thus supporting the necessity of careful and product-oriented choice of tobacco varieties for the production in each region.

Table 4. Complex evaluation of the FCV tobacco varieties from South and North Bulgaria regions

\begin{tabular}{|c|c|c|c|c|c|c|c|c|c|c|c|}
\hline \multirow{3}{*}{ Index } & \multicolumn{5}{|c|}{ Ranking of the varieties } & \multirow{3}{*}{$\mathrm{CI}^{6}$} & \multicolumn{5}{|c|}{ Quality index } \\
\hline & \multirow{2}{*}{$\begin{array}{l}\text { PVH19 } \\
\text { PLV }^{3} \\
\end{array}$} & \multirow{2}{*}{$\begin{array}{l}\text { H9 } \\
\text { PLV }\end{array}$} & \multirow{2}{*}{$\begin{array}{l}\text { PVH19 } \\
\text { PRM }^{4}\end{array}$} & $\mathbf{V}$ & Niki & & \multirow{2}{*}{$\begin{array}{c}\text { PVH19 } \\
\text { PLV }\end{array}$} & \multirow{2}{*}{$\begin{array}{l}\text { H9 } \\
\text { PLV }\end{array}$} & \multirow{2}{*}{$\begin{array}{c}\text { PVH19 } \\
\text { PRM }\end{array}$} & \multirow{2}{*}{\multicolumn{2}{|c|}{\begin{tabular}{c|c}
$\mathbf{V}$ & Niki \\
CNB
\end{tabular}}} \\
\hline & & & & \multicolumn{2}{|c|}{$\mathbf{C N B}^{5}$} & & & & & & \\
\hline Nicotine (\%) & 2 & 1 & 3 & 4.5 & 4.5 & 0.20 & 0.40 & 0.20 & 0.60 & 0.90 & 0.90 \\
\hline $\mathrm{TN} / \mathrm{Nic}^{1}$ & 1 & 3.5 & 5 & 2 & 3.5 & 0.18 & 0.18 & 0.63 & 0.90 & 0.36 & 0.63 \\
\hline $\mathrm{RS} / \mathrm{Nic}^{2}$ & 1.5 & 1.5 & 3 & 4.5 & 4.5 & 0.12 & 0.18 & 0.18 & 0.36 & 0.54 & 0.54 \\
\hline Tar (mg/cig) & 4.5 & 3 & 4.5 & 1.5 & 1.5 & 0.10 & 0.45 & 0.30 & 0.45 & 0.15 & 0.15 \\
\hline Expert assessment & 5 & 1 & 2 & 3 & 4 & 0.15 & 0.75 & 0.15 & 0.30 & 0.45 & 0.60 \\
\hline Smoking assessment & 1 & 2 & 3 & 4 & 5 & 0.25 & 0.25 & 0.50 & 0.75 & 1.00 & 1.25 \\
\hline \multicolumn{7}{|c|}{ Quality index score } & 2.21 & 1.96 & 3.36 & 3.40 & 4.07 \\
\hline \multicolumn{7}{|c|}{ Rating of the varieties } & 2 & 1 & 3.5 & \begin{tabular}{|l|}
3.5 \\
\end{tabular} & 5 \\
\hline
\end{tabular}

${ }^{1} \mathrm{TN} / \mathrm{Nic}$ - total nitrogen/nicotine; ${ }^{2} \mathrm{RS} / \mathrm{Nic}$ - reducing sugars/nicotine; ${ }^{3} \mathrm{PLV}$ - region Plovdiv; ${ }^{4} \mathrm{PVM}$ - region Parvomay; ${ }^{5} \mathrm{CNB}$ region Central North Bulgaria; ${ }^{6} \mathrm{CI}$ - coefficient of importance (relative weight).

The obtained results (Table 4) provided yet another proof in favor of the assumption that the complex evaluation of tobacco varieties, which takes into consideration all major quality-related indices and their relative importance, is a prerequisite for making responsible decisions connected to variety choice, on a national or regional basis, as there was no uniformity in varieties' quality profiles with regard to a single index (Tables 1, 2 and 3). 


\section{Conclusions}

The results from the investigation indicated specific differences in the quality-related indices of the varieties, based on the comparison of FCV tobaccos from the two production areas (South and North Bulgaria) and between the varieties within each of them.

The local PVH19 variety from the region of Plovdiv significantly outclassed the rest of the varieties by chemical indices, being close to the "typical" FCV tobacco profile, with higher leaf and smoke nicotine content $(1.77 \%$ and $1.43 \mathrm{mg} / \mathrm{cig}$, respectively) and balanced total nitrogen/nicotine (0.83) and reducing sugars/nicotine (10.96) ratios.

The expert assessment of leaf quality elements, important in tobacco grading and selling, revealed significant differences between the varieties; the best rated in that test was the introduced $\mathrm{H} 9$ variety (origin: Hungary) from the region of Plovdiv, followed by the local PVH19 variety from the region of Parvomay and the two varieties from the Central North Bulgaria region (V and Niki), while the PVH19 variety from the region of Plovdiv was ranked the last.

The smoking profiles of the studied varieties showed smaller variation between the local and the introduced varieties within the regions; still, the tobaccos from the South Bulgaria regions were rated better than those from the North Bulgaria area. Individually, the best smoking profile attributes were found in the mass-produced local PVH19 variety from Plovdiv region and in the introduced $\mathrm{V}$ variety (origin: Greece) from Central North Bulgaria region.

The complex evaluation of the studied FCV tobacco varieties, which took into consideration the results from the chemical, expert and smoking assessments and their relative importance, provided the final rating of the tobaccos, based on the quality index scores. In that rating order, the best evaluated was the H9 variety introduced for the region of Plovdiv, followed closely by the local PVH19 variety from the same region; the next were the local PVH19 (Parvomay) and the introduced V (Central North Bulgaria) varieties, with identical quality indices, while the other local variety, Niki (Central North Bulgaria region), was assigned the last rank.

Therefore, the multistep assessment of local (with a history of production) and introduced FCV tobacco varieties from different regions of Bulgaria revealed some important aspects of tobacco quality formation, which might be of practical relevance to the production, processing and realization of FCV tobacco varieties on a regional and national basis.

\section{References}

1. E. Nikolov, T. Hristeva, V. Masheva, Sci. Works Agric. Uni. - Plovdiv. 50, 143-149 (2005)

2. E. Nikolov, T. Hristeva, V. Masheva, Tobacco. 55, 189-193 (2005)

3. K. Kocoska, I. Risteski, Tobacco. 61, 3-9 (2011)

4. Y. Dyulgerski, Tobacco. 64, 13-18 (2014)

5. Y. Dyulgerski, Acta Agric. Ser. 19, 61-69 (2014)

6. D. Drachev, V. Nikolova, V. Popova, Sustainable Economic Development of Regions, (East West Association, Vienna, 2015)

7. G. Regassa, B. S. Chandravanshi, Springer Plus. 5, 232 (2016)

8. X. Qiao, B. Wang, B. Xiong, Y. Ma, Z. Gao, R. Zhao, X. Liu, S. Dong, H. Xu, Q. Liu, D. Luo, Tob. Sci. Technol. 50, 66-72 (2017)

9. M. Bortolini, M. Gamberi, C. Mora, A. Regattieri, Int. J. Green Ener. 16, 1220-1229 (2019)

10. R. Bozhinova, Bulg. J. Soil Sci., Agrochem. Ecol. 53, 1724 (2019)

11. T. Hristeva, Bulg. J. Crop Sci., 2, 175-178 (2009)

12. L. P. C. Tedesco, A. C. de Freitas, R. F. Molz, J. N. C. Schreiber, Rev. Bras. Eng. Agric. Ambient. 23, 782-786 (2019)

13. Tobacco and Tobacco Products Institute, Instruction for the harvesting and curing of tobacco from Virginia variety group (Ministry of Agriculture, Food and Forestry, Sofia, 2016)

14. North Carolina State University. Flue-cured tobacco guide (NC State Extension, Raleigh, NC, 2021)

15. ISO 15152: 2003. Tobacco. Determination of the content of total alkaloids as nicotine - continuous-flow analysis method (ISO, Geneva, 2003)

16. ISO 15154:2003, Tobacco. Determination of the content of reducing carbohydrates - continuous-flow analysis method (ISO, Geneva, 2003)

17. BDS 15836:1988, Tobacco and tobacco products. Methods of total nitrogen determination (BIS, Sofia, 1988)

18. ISO 2817:1999, Tobacco and tobacco products. Determination of silicated residues insoluble in hydrochloric acid (ISO, Geneva, 1999)

19. BDS 17365:1994, Tobacco and tobacco products. Determination of heavy metals and arsenic content. Preparation methods (BIS, Sofia, 1994)

20. S. Gueorgiev, V. Popova, Biotechnol. Biotechnol. Equip. 1, 61-65 (1999)

21. Drachev D., V. Nikolova, N. Nikolov, Biotechnol. Biotechnol. Equip. 19, 192-201 (2005)

22. Nikolova V. Bulg. J. Agric. Sci. 13, 657-671 (2007)

23. BDS 11750:1985, Tobacco and tobacco products. Bulgarian Virginia tobacco, manipulated and fermented. (BIS, Sofia, 1985)

24. V. P. Borovikov, I.P. Borovikov, Statistica. Statistical analysis and data processing in the Windows environment (Filin, Moscow, 1998)

25. T. C. Tso, Production, physiology and biochemistry of tobacco plant (Ideals, Nashville, TN, 1991) 